\title{
Reconsidering the use of the general linear model with single-case data
}

\author{
JOHN FERRON \\ University of South Florida, Tampa, Florida
}

\begin{abstract}
Using a low point estimate of autocorrelation to justify analyzing single-case data with the general linear model (GLM) is questioned. Monte Carlo methods are used to examine the degree to which bias in the estimate of autocorrelation depends on the complexity of the linear model used to describe the data. A method is then illustrated for determining the range of autocorrelation parameters that could reasonably have led to the observed autocorrelation. The argument for using a GLM analysis can be strengthened when the GLM analysis functions appropriately across the range of plausible autocorrelations. For situations in which the GLM analysis does not function appropriately across this range, a method is provided for adjusting the confidence intervals to ensure adequate coverage probabilities for specified levels of autocorrelation.
\end{abstract}

Using some form of the general linear model (GLM) to analyze single-case data has been considered for decades. In the early seventies, several procedures based on the analysis of variance were suggested (Gentile, Roden, \& Klein, 1972; Shine \& Bower, 1971), and more recently, regression procedures have been illustrated (Center, Skiba, \& Casey, 1986; Huitema \& McKean, 1998). Reservations about using GLM-based methods have also been expressed and tend to hinge on concerns about violating the assumption of independently distributed errors (e.g., Kratochwill et al., 1974; Thoresen \& Elashoff, 1974; Toothaker, Banz, Noble, Camp, \& Davis, 1983).

The errors are influenced by effects that are not considered in the model. For example, the discrepancy between a child's observed level of behavior and the level predicted by the model may be partially attributable to the fact the child was ill on the day the measurement took place. In single-case data, the potential exists for the nonmodeled effects, such as the effect of being ill, to influence multiple consecutive observations in the series. This in turn may lead to similarity among errors that are close to each other in time. If so, the errors would be positively autocorrelated, since the errors at time $t, e_{t}$, would be positively correlated with the errors at time $t-1, e_{t-1}$.

The effects of positive autocorrelation on GLM-based methods has been well documented. In general, we would expect the regression coefficients to be unbiased but the standard errors of the regression coefficients to be underestimated (Neter, Wasserman, \& Kutner, 1990). This in turn leads to confidence intervals that tend to be too small

I thank the anonymous reviewers for their comments on an earlier version of this article. Correspondence concerning this article should be addressed to J. Ferron, University of South Florida, 4202 East Fowler Ave., EDU 162, Tampa, FL 33620 (e-mail: ferron @tempest.coedu. usf.edu). and significance tests with inflated Type I error rates. The degree to which confidence intervals and significance tests are impacted increases with the level of autocorrelation and has been documented for various series lengths and patterns of autocorrelation (Greenwood \& Matyas, 1990; Huitema, McKean, \& McKnight, 1999; Scheffé, 1959; Toothaker et al., 1983). There appears to be agreement that positive autocorrelation can have serious effects on GLM-based analyses but much less agreement as to the amount of autocorrelation that typically underlies the errors in single-case data (e.g., Busk \& Marascuilo, 1988; Huitema, 1985; Matyas \& Greenwood, 1997).

Recently, Huitema and McKean (1998) have argued that past estimates of autocorrelation have often been based on observed values of the outcome, instead of on errors. This has led to overestimates of the degree of autocorrelation and a premature dismissal of the use of GLM-based methods. They go on to illustrate a modeling strategy for singlecase data that involves a preliminary test for autocorrelation. Autocorrelation is estimated for the errors of a reasonable model of the data. If this autocorrelation estimate is low and not statistically significant, it is assumed that the degree of autocorrelation does not raise problems and that the confidence intervals and statistical tests can be interpreted in their usual manner.

This approach is generally consistent with the long-standing practice of screening data for violations of assumptions prior to conducting analyses, consistent with screening time series data for autocorrelation (Durbin \& Watson, 1951) and consistent with Hartmann's (1974) suggestion to screen single-case data for violations of the independence assumption. The data-screening procedure illustrated by Huitema and McKean (1998) involves a null hypothesis test for autocorrelation. Of course, the test for autocorrelation also has assumptions, and to test these assumptions, more assumptions would need to be made and tested. 
In addition, tests for autocorrelation have relatively low power for series lengths typically encountered in behavioral research (L'Esperance \& Taylor, 1975; Suen \& Ary, 1987). Consequently, the interpretation of not statistically significant as not consequential is suspect. The justification for using GLM could be strengthened, however, by showing that nontrivial autocorrelation parameters are unlikely to lead to autocorrelation estimates as low as the one observed.

A method will be illustrated for determining the range of autocorrelation parameters that could reasonably have led to the observed autocorrelation. A method will then be provided for determining the degree to which a GLM analysis functions appropriately across this range. If the GLM analysis functions appropriately across the range of plausible autocorrelations, the argument for proceeding with a GLM analysis will be strengthened. For situations in which the analysis does not function appropriately across this range, it should be acknowledged that there is uncertainty in the autocorrelation and, thus, uncertainty in the standard errors of the GLM analysis. For these cases, a method will be provided for adjusting the alpha to ensure adequate coverage probabilities for specified levels of autocorrelation.

\section{Bias in Estimating Autocorrelation}

The most common estimator of autocorrelation is

$$
r_{1}=\frac{\sum_{t=2}^{N} e_{t} e_{t-1}}{\sum_{t=1}^{N} e_{t}^{2}},
$$

where $e_{t}$ is the error at time $t, e_{t-1}$ is the error at time $t-1$, and $N$ is the number of observations in the series. This estimator is biased (Huitema \& McKean, 1991; Marriot \& Pope, 1954; Matyas \& Greenwood, 1991). Theoretical expressions for the bias are available for some models. For example, if the errors are generated from a first-order autoregressive model, $e_{t}=\rho_{1} e_{t-1}+a_{t}$, and the data are generated from a mean model, $Y_{t}=\mu+e_{t}$, then the bias is approximately $-2 \rho_{1} / N$ if $\mu$ is known and approximately $-\left(1+3 \rho_{1}\right) / N$ if $\mu$ has to be estimated (Marriott \& Pope, 1954). The size of the bias depends not only on the amount of autocorrelation, $\rho_{1}$, and the series length, $N$, but also on whether or not the mean has to be estimated. To illustrate the effect of estimating the mean, consider a series length of 20 and an autocorrelation of .3. If the mean is known, the expected value of $r_{1}$ is approximately .27 , but if the mean is estimated, the expected value of $r_{1}$ is approximately 205 .

One may anticipate that the bias in the autocorrelation estimate would be more substantial when the errors of complex intervention models requiring estimation of the mean and the slope for multiple phases are used. Huitema and McKean (2000b) show that when the true autocorrelation is zero, the bias is approximately $-P / N$, where $P$ is the number of parameters in the regression model. When the true autocorrelation is not zero, the amount of bias is more questionable.

To explore the impact of model complexity on bias, a small set of simulations was run using the ARMASIM function in PROC IML in SAS (SAS Institute, 1996). One hundred thousand series were generated of length 20 , using a first-order autoregressive model with the autocorrelation parameter set to .3. Eight regression models were estimated for each series, and the errors of each of these models were used to estimate autocorrelation. The distribution of the autocorrelation estimates was then examined for each of the eight models.

For the first three models, the series was conceptualized as a single phase. In the first model, the known mean was used; in the second model, the mean was estimated; and in the third model, an intercept and slope were estimated. In the fourth, fifth, and sixth models, the series was conceptualized to have arisen from an $\mathrm{AB}$ design with equal phase lengths. In the fourth model, the mean was estimated for both phases; in the fifth model, the level for the first phase, the level for the second phase, and a common slope were estimated; and in the sixth model, the intercept and the slope were estimated for each phase. For the last two models, the series was modeled as if it had come from an ABAB design with equal phase lengths. The seventh model involved the estimation of the mean of each of the four phases, and the eighth model involved the estimation of a separate intercept and slope for each of the four phases.

The sampling distribution of $r_{1}$ for each of the eight models is shown in Figure 1, using modified boxplots showing the 5 th, 25 th, 50 th, 75 th, and 95 th percentiles. For the first two models, the center of the sampling distribution is very close to the expected value of $r_{1}$ based on theoretical expressions (i.e., .27 and .205, respectively). Note that the numbers of regression coefficients estimated in the eight models were zero, one, two, two, three, four, four, and eight, respectively. For models with the same number of estimated coefficients, the distributions are extremely similar. For models involving greater numbers of estimated regression coefficients, the bias in the autocorrelation estimator is substantially larger. As the number of estimated parameters increases from zero to four to eight, the median of the sampling distribution of $r_{1}$ moves from .27 to -.03 to -.33 .

The distributions of $r_{1}$ displayed in Figure 1 are based on a series length of 20 and an autocorrelation parameter, $\rho_{1}$, of .30. The bias in $r_{1}$ becomes greater when the series length is shortened and $\rho_{1}$ is increased. A SAS IML program is provided in Appendix A that will allow a researcher to explore the amount of bias in $r_{1}$ for a given level of autocorrelation (e.g., $\rho_{1}=.20$ ) and a particular single-case design (e.g., a 30 observation $\mathrm{AB}$ design in which the intervention leads to an immediate change in level). This program can also be used to determine a range of plausible values for the autocorrelation parameter. 


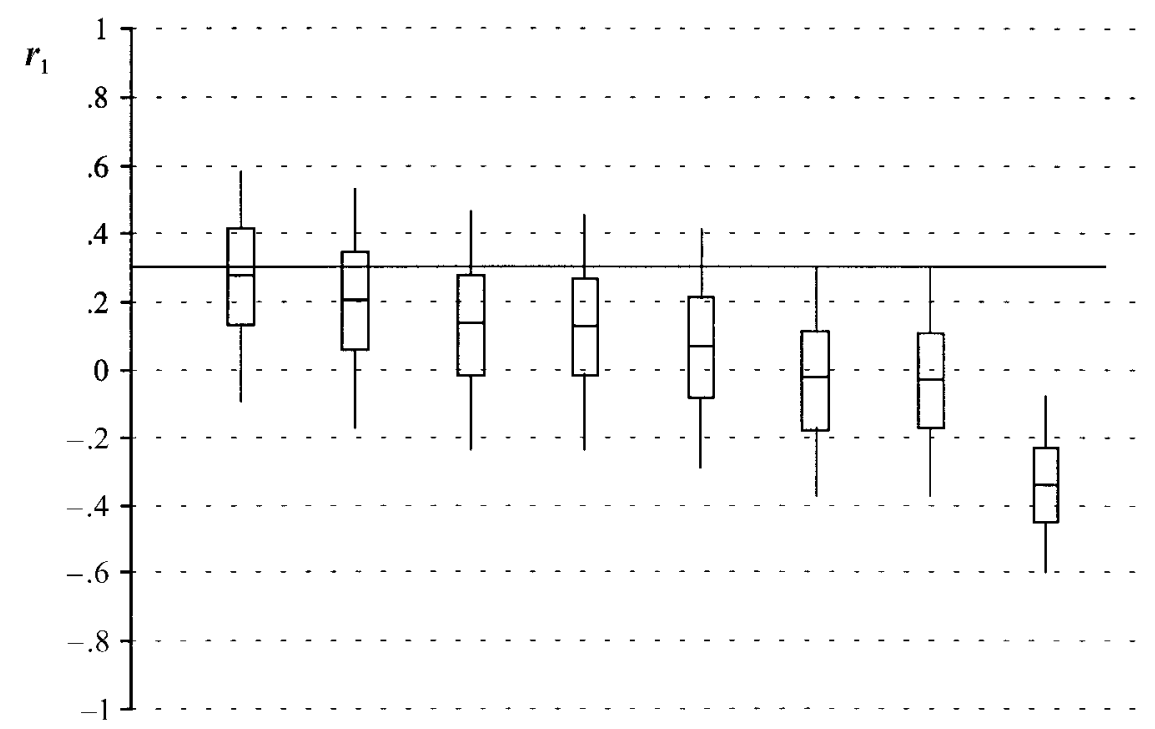

Model 1 Model 2 Model 3 Model 4 Model 5 Model 6 Model 7 Model 8

Figure 1. Sampling distribution of $r_{1}$ as a function of model complexity for $\rho_{1}=.3$ and series length $=20$. Boxplots show the 5 th, 25 th, 50 th, 75 th, and 95 th percentiles.

\section{Determining Plausible Values for the Autocorrelation Parameter}

By running simulations focusing on a single intervention model and a variety of autocorrelation values, one may find a range of plausible values for the autocorrelation parameter. To use the SAS IML program provided in Appendix $A$, the researcher supplies the number of series to generate (e.g., rep $=100000$ ), the level of autocorrelation (e.g., phi $=\{1-.3\}$ ), the design matrix (e.g., $\mathbf{x}=$ $\{110,120, \ldots, 1101\})$, and the regression parameters (e.g., beta $=\{0,0,0\}$ ). Note that the sign of the autocorrelation parameter must be reversed. Setting phi equal to $\{1-.3\}$ results in a first-order autoregressive model with an autocorrelation of positive .3. It should also be noted that the researcher need not be too concerned with the actual size of the regression parameters, since the values of the beta matrix do not influence the distribution of $r_{1}$. For simple models, this can be seen in the theoretical expressions for bias and variability (Marriott \& Pope, 1954). For more complex models, it can be demonstrated by altering the values of beta in the program provided.

The design matrix shown is appropriate for an $\mathrm{AB}$ design with five observations per phase, where the data can be described using a common slope and a shift in levels between phases. Other single-case designs can be examined by modifying the $\mathbf{x}$ matrix. Huitema and McKean (2000a) provide a description of various design matrices appropriate for single-case research. Of course, a change in the number of columns in $\mathbf{x}$ must be accompanied by a corresponding change in the number of rows in beta.

As an example, consider an $\mathrm{AB}$ design with 40 observations, 20 per phase, and a treatment effect leading to a change in level and slope. Suppose further that a sample autocorrelation of 0.0 is observed for the errors of the model. To get a sense of the range of autocorrelation parameters that may have given rise to this estimate, the errors for 100,000 series were generated with a first-order autoregressive model with autocorrelation parameters of $0, .1, .2, .3, .4$, and .5 . The distribution of $r_{1}$ values that resulted for each level of $\rho_{1}$ are presented in Figure 2 .

Five percent of each distribution falls below the bottom of the lower whisker. If the autocorrelation parameter were .4, one would expect to see estimated autocorrelation values of zero or less more than $5 \%$ of the time. Thus, using $5 \%$ as a criterion for unlikely, it would be difficult to conclude that an autocorrelation estimate as low as 0.0 is unlikely given an autocorrelation parameter of .4. It could be concluded, however, that an autocorrelation estimate of 0.0 is unlikely if the autocorrelation parameter is .5. By running the program allowing the values of $\rho_{1}$ to vary between .4 and .5 in increments of .01 , it was determined that .46 was the largest value of $\rho_{1}$ for which the estimated autocorrelation would fall below the obtained estimate of 0.0 over $5 \%$ of the time.

For this example, there is a wide range of plausible values for the autocorrelation parameter. The value of $\rho_{1}$ may be 0 , but it may be as high as .46. This uncertainty in the size of the autocorrelation parameter should translate into uncertainty about the functioning of the GLM analysis. More particularly, the size of the standard errors for the regression coefficients should be questioned, which leads to concerns about coverage probabilities and the rate of Type I errors. Note that the above examination focused on plausible positive values for the autocorrelation parameter, since positive values seem more credible in social science settings and are known to lead to underestimates of 


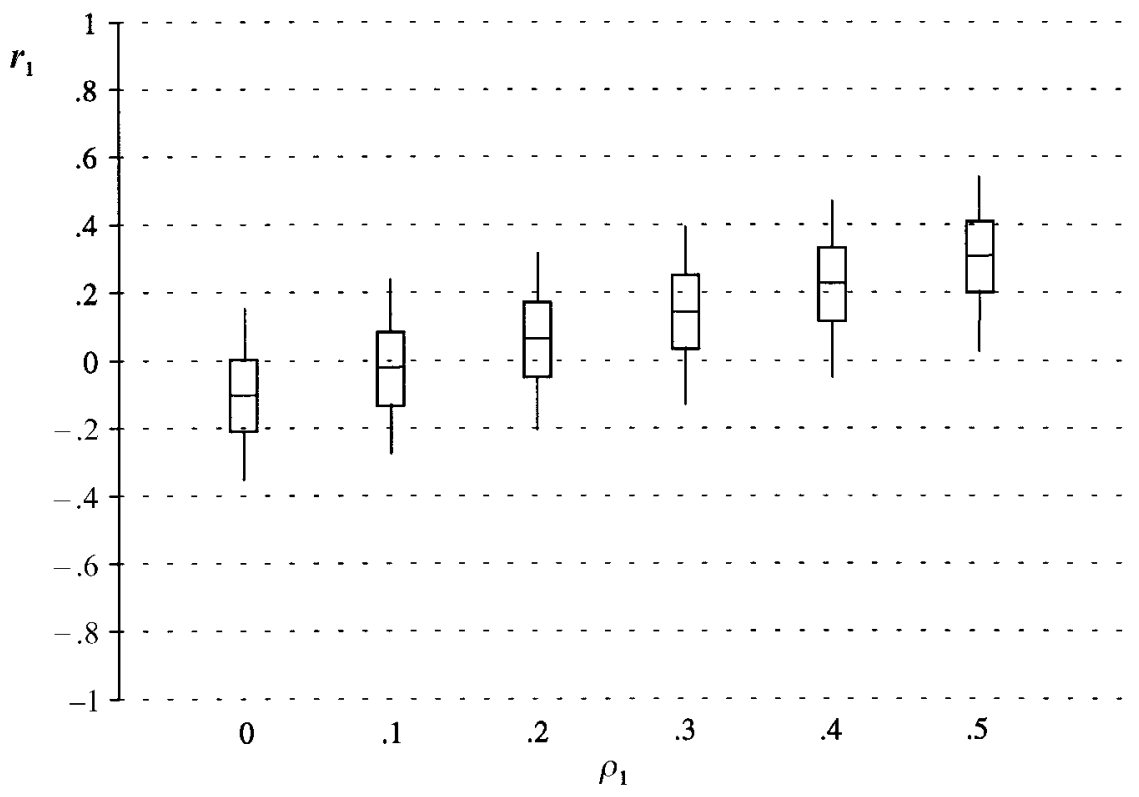

Figure 2. Sampling distribution of $r_{1}$ as a function of autocorrelation for a 40-observation AB design leading to a change in level and a change in slope. Boxplots show the 5th, 25th, 50th, 75th, and 95th percentiles.

the standard errors of a GLM analysis. One could, if desired, also use the program to explore negative values of autocorrelation.

\section{Exploring the Effect of Autocorrelation on GLM Analyses}

Effects of autocorrelation on GLM analyses have been documented for some situations (Greenwood \& Matyas, 1990; Huitema et al., 1999; Scheffé, 1959; Toothaker et al., 1983). The effects are not always predicted well by asymptotic theory and depend on sample size, the complexity of the design, and the type of parameter being considered (Huitema et al., 1999). Since researchers may find themselves in situations that do not match those that have been studied, it may be useful for researchers to have a program that allows them to look at coverage probabilities and Type I error rates for a particular design and level of autocorrelation. Such a program is provided in Appendix B. The user provides a nominal alpha level (e.g., alpha $=.05)$, the number of simulated experiments (e.g., rep $=100000$ ), degree of autocorrelation (e.g., phi $=$

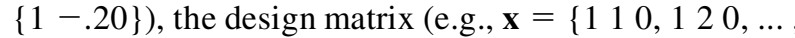
$1101\}$ ), and the matrix of regression parameters (e.g., beta $=\{0,0,0\})$. The program can be run with different values of $\rho_{1}$ to get a sense of the impact of various levels of autocorrelation.

Let us again consider the $\mathrm{AB}$ design with 20 observations per phase, a change in level and slope, and an estimated autocorrelation of 0.0 . Three values of the autocorrelation parameter are considered. A value of 0.0 , which represents no autocorrelation, a value of .12, which is the value that leads to a sampling distribution with a median equal to the observed autocorrelation, and a value of .46, which is the largest value having a sampling distribution with at least $5 \%$ of the values below the observed autocorrelation.

Consider the regression coefficient indexing a shift in level. If the autocorrelation parameter were zero and the nominal alpha were set to .05 , the coverage probability would be .95 , and the Type I error rate would be .05 . If $\rho_{1}$ were .12 , the coverage probability would be estimated to be .920 , using the program in Appendix B and the Type I error rate would be estimated to be .080 . This exceeds Bradley's (1978) liberal criteria for judging the robustness of statistical tests. If $\rho_{1}$ were .46 , the coverage probability would be estimated to be .769 , and the Type I error rate would be estimated to be .231 . Clearly, the GLM analysis is not robust across all the plausible levels of autocorrelation in this example.

\section{Adjusting Alpha to Compensate for Autocorrelation}

In situations in which a researcher cannot argue that the GLM analysis is robust across the entire range of plausible autocorrelations, the researcher may wish to consider altering the analysis on the basis of the observed level of autocorrelation. An analysis that is altered based on the observed data is sometime referred to as adaptive (Hogg, 1974; Zumbo, 2000). Zumbo presents an adaptive analysis for coupled data that has some parallels with the analysis that will be illustrated here. In his analysis, a $90 \%$ confidence interval was constructed for the intercouple 
correlation. A $t$ test for mean differences was then computed for different plausible values for the intercouple correlation.

Here, confidence intervals for the treatment effect will be constructed for a variety of plausible values of the autocorrelation parameter. To construct the confidence intervals, an alpha adjustment method is used. By systematically varying the value of alpha in the program in Appendix B, an alpha can be found that ensures an adequate coverage probability for a given level of autocorrelation. By repeating this process for a variety of plausible levels of autocorrelation, a range of intervals is produced. If the largest of these intervals contains only values judged to be of practical importance, arguments regarding the effectiveness of the treatment could be strengthened. The arguments would be stronger than they would be if they were based only on a confidence interval assuming no autocorrelation and stronger than they would be if they were based simply on the point estimate of the treatment effect.

To illustrate, consider the data analyzed by Huitema and McKean (1998) that were part of a study examining the effects of feedback on personal appearance published by McClannahan, McGee, MacDuff, and Krantz (1990). The original study used a multiple baseline design involving data collected from three homes. Data from the first home will be considered here. The data from this home consists of a series of 21 observations, 6 before treatment and 15 after treatment. Huitema and McKean (1998) found a model allowing for a common slope, but a shift in level was reasonable for the data, $Y_{t}=\beta_{0}+\beta_{1} t+$ $\beta_{2} D_{t}+e_{t}$. The shift in level was estimated to be 8.69 points on the appearance measure.

The estimate of $r_{1}$ based on the errors of this model was - .37. By using the program in Appendix A, it was determined that a $\rho_{1}$ of .15 was the largest plausible level of autocorrelation for a first-order autoregressive model. The effect of this level of autocorrelation was examined by using the program in Appendix B. If $\rho_{1}$ is equal to .15, only $92.1 \%$ of traditionally computed $95 \%$ confidence intervals would contain $\beta_{2}$, and the Type I error rate for testing $\beta_{2}$ would be .079 . Thus, the GLM does not function appropriately across the range of plausible autocorrelations.

If one assumes that $\rho_{1}$ equals zero, the $95 \%$ confidence interval is $(2.16,15.21)$. If one assumes a worse case scenario, $\rho_{1}=.15$, the $95 \%$ confidence interval will be larger. By varying the alpha in the program in Appendix B, it was found that an adjusted alpha of .029 would lead to intervals containing $\beta_{2} 95 \%$ of the time, when $\rho_{1}$ equals .15 and the errors follow a first-order autoregressive model. When this alpha is used, the more conservative confidence interval would be estimated as $(1.31,16.06)$. Thus, the conclusion that feedback led to a positive change in appearance can be supported not only if the errors are independent, but also if the errors follow a first-order autoregressive model with the maximum plausible level of autocorrelation.

The effect of feedback on appearance was also considered by assuming a first-order moving average model, $e_{t}=$ $a_{t}-\theta_{1} a_{t-1}$. It was determined that the largest plausible magnitude for a negative moving average parameter was .13 when the program in Appendix A was used. Negative moving average parameters were considered, since they result in positive levels of autocorrelation. Again, SAS notation requires the entered sign to be opposite that of conventional notation. To obtain a moving average parameter of -.13 , one sets theta equal to $\{1.13\}$.

By using the program from Appendix B, it was found that if the errors were generated by a first-order moving average model with a moving average parameter of -.13 , about $92.7 \%$ of confidence intervals calculated under the independence assumption would contain $\beta_{2}$. This is slightly better coverage than the $92.1 \%$ estimate obtained when we considered the highest plausible level of autocorrelation obtained using a first-order autoregressive model. The alpha adjustment needed for the worst case autoregressive model is larger than the adjustment needed for the worst case moving average model. Thus, the confidence interval for the autoregressive model is wider than that of the moving average model. The conclusion that there was a positive treatment effect holds whether one assumes an independent error structure, a first-order autoregressive structure, or a first-order moving average structure.

\section{Discussion}

Researchers should not proceed with a GLM analysis of single-case data without carefully examining the errors of the model. A low estimated level of autocorrelation is not sufficient evidence for concluding that the standard errors of a GLM analysis are accurate. One should consider alternative models that could have given rise to the observed estimate. When multiple models (e.g., $e_{t}=.07 e_{t-1}+a_{t}$, $e_{t}=.31 e_{t-1}+a_{t}$, and $\left.e_{t}=.18 e_{t-1}+a_{t}\right)$ could have reasonably led to the observed errors, the researcher should acknowledge uncertainty in the level of autocorrelation. This uncertainty should result in uncertainty in the size of the standard errors and, thus, uncertainty in the size of the confidence intervals.

In these situations, researchers may wish to consider alternatives to a GLM analysis. An adaptive analysis was illustrated that allows the researcher to create confidence intervals for a variety of plausible error models. This provides a way to explore and index the consequences of assuming various degrees of autocorrelation. Other alternatives include visual analyses (Parsonson \& Baer, 1986), effect sizes (Kromrey \& Foster-Johnson, 1996), randomization tests (Edgington, 1980; Ferron \& Sentovich, 2002; Koehler \& Levin, 1998), integrated moving average (ARIMA) intervention models (Box \& Tio, 1975), and a double bootstrap method (McKnight, McKean, \& Huitema, 2000).

\section{REFERENCES}

Box, G. E. P., \& Tro, G. C. (1975). Intervention analysis with applications to economic and environmental problems. Journal of the American Statistical Association, 70, 70-79.

BRADLEY, J. C. (1978). Robustness? British Journal of Mathematical \& Statistical Psychology, 31, 144-152.

Busk, P. L., \& Marascuilo, L. A. (1988). Autocorrelation in single- 
subject research: A counterargument to the myth of no autocorrelation. Behavioral Assessment, 10, 229-242.

Center, B. A., Skiba, R. J., \& CASey, A. (1986). A methodology for the quantitative synthesis of intra-subject design research. Journal of Special Education, 19, 387-400.

Durbin, J., \& WATsOn, G. S. (1951). Testing for serial correlation in least squares regression: II. Biometrika, 38, 159-178.

EDGINGTON, E. S. (1980). Validity of randomization tests for one-subject experiments. Journal of Educational Statistics, 5, 235-251.

Ferron, J., \& Sentovich, C. (2002). Statistical power of randomization tests used with multiple-baseline designs. Journal of Experimental Education, 70, 165-178.

Gentile, J. R., Roden, A. H., \& Klein, R. D. (1972). An analysis-ofvariance model for the intrasubject replication design. Journal of Applied Behavior Analysis, 5, 193-198.

Greenwood, K. M., \& Matyas, T. A. (1990). Problems with the application of interrupted time series analysis for brief single-subject data Behavioral Assessment, 12, 355-370.

Hartmann, D. P. (1974). Forcing square pegs into round holes: Some comments on "an analysis-of-variance model for the intrasubject replication design.” Journal of Applied Behavior Analysis, 7, 635-638.

HogG, R. V. (1974). Adaptive robust procedures: A partial review and some suggestions for future applications and theory. Journal of the American Statistical Association, 69, 909-923.

Huitema, B. E. (1985). Autocorrelation in applied behavior analysis: A myth. Behavioral Assessment, 7, 107-118.

Huitema, B. E., \& McKean, J. W. (1991). Autocorrelation estimation and inference with small samples. Psychological Bulletin, 110, 291304.

Huitema, B. E., \& McKean, J. W. (1998). Irrelevant autocorrelation in least-squares intervention models. Psychological Methods, 3, 104 116.

Huitema, B. E., \& McKean, J. W. (2000a). Design specification issues in time-series intervention models. Educational \& Psychological Measurement, 60, 38-58.

Huitema, B. E., \& McKean, J. W. (2000b). A simple and powerful test for autocorrelated errors in OLS intervention models. Psychological Reports, 87, 3-20.

Huitema, B. E., McKean, J. W., \& McKnight, S. (1999). Autocorrelation effects on least-squares intervention analysis of short time series. Educational \& Psychological Measurement, 59, 767-786.

Koehler, M. J., \& LeVIN, J. R. (1998). Regulated randomization: A potentially sharper analytical tool for the multiple-baseline design. Psychological Methods, 2, 206-217.

Kratochwill, T., Alden, K., Demuth, D., Dawson, D., Panicucci, C., Arntson, P., McMurray, N., Hempstead, J., \& Levin, J. (1974). A further consideration in the application of an analysis-of-variance model for the intrasubject replication design. Journal of Applied Behavior Analysis, 7, 629-633.

Kromrey, J. D., \& Foster-Johnson, L. (1996). Determining the efficacy of intervention: The use of effect sizes for data analysis in singlesubject research. Journal of Experimental Education, 65, 73-93.

L'ESPERANCE, W. L., \& TAYLOR, D. (1975). The power of four tests of autocorrelation in the linear regression model. Journal of Econometrics, 3, 1-21.

Marriott, F. H. C., \& Pope, J. A. (1954). Bias in the estimation of autocorrelations. Biometrika, 41, 390-402.

Matyas, T. A., \& Greenwood, K. M. (1991). Problems in the estimation of autocorrelation in brief time series and some implications for behavioral data. Behavioral Assessment, 13, 137-157.

Matyas, T. A., \& Greenwood, K. M. (1997). Serial dependency in singlecase time series. In R. D. Franklin, D. B. Allison, \& B. S. Gorman (Eds.), Design and analysis of single-case research (pp. 215-243). Mahwah, NJ: Erlbaum.

McClannahan, L. E., McGee, G. G., MacDuff, G. S., \& Krantz, P. J. (1990). Assessing and improving child care: A personal appearance index for children with autism. Journal of Applied Behavior Analysis, 23, 469-482.

McKnight, S. D., McKean, J. W., \& Huitema, B. E. (2000). A double bootstrap method to analyze linear models with autoregressive error terms. Psychological Methods, 5, 87-101.

Neter, J., Wassermann, W., \& Kutner, M. H. (1990). Applied linear statistical models: Regression, analysis of variance, and experimental designs (3rd ed.). Homewood, IL: Irwin.

PARsonson, B. S., \& BAER, D. M. (1986). The graphic analysis of data. In A. Poling \& R. W. Fuqua (Eds.), Research methods in applied behavior analysis: Issues and advances (pp. 157-186). New York: Plenum Press. SAS Institute (1996). SAS, Version 6.12 [Computer program]. Carey, NC: Author.

SCHEFFÉ, H. (1959). The analysis of variance. New York: Wiley.

SHINe, L. C., \& Bower, S. M. (1971). A one-way analysis of variance for single-subject designs. Educational \& Psychological Measurement, 31, 105-113.

SuEN, H. K., \& ARY, D. (1987). Autocorrelation in applied behavior analysis: Myth or reality? Behavioral Assessment, 9, 125-130.

Thoresen, C. E., \& ElAShoff, J. D. (1974). "An analysis-of-variance model for intrasubject replication design": Some additional comments. Journal of Applied Behavior Analysis, 7, 639-641.

Toothaker, L. E., Banz, M., Noble, C., Camp, J., \& Davis, D. (1983). $N=1$ designs: The failure of ANOVA-based tests. Journal of Educational Statistics, 4, 289-309.

Zumbo, B. D. (2000, April). Adaptive inference. Paper presented at the meeting of the American Educational Research Association, New Orleans. 
APPENDIX A

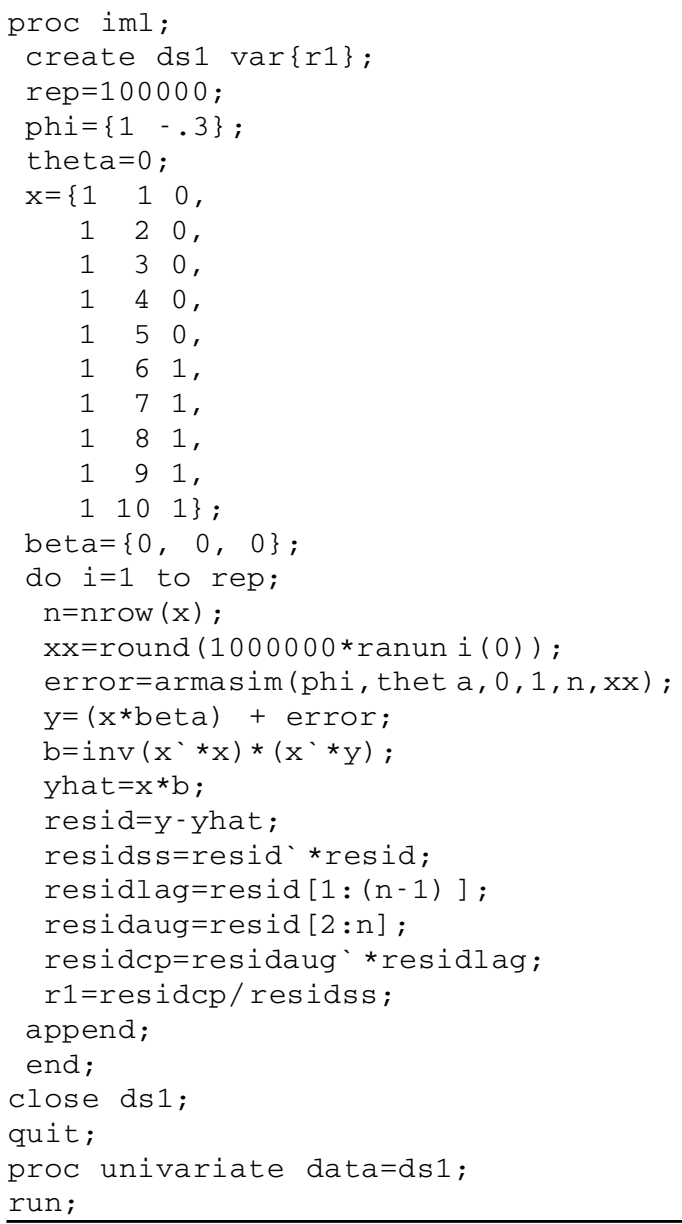

APPENDIX B

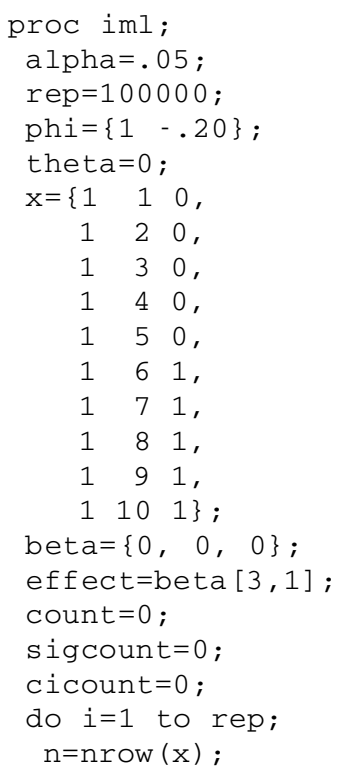


APPENDIX B (Continued)

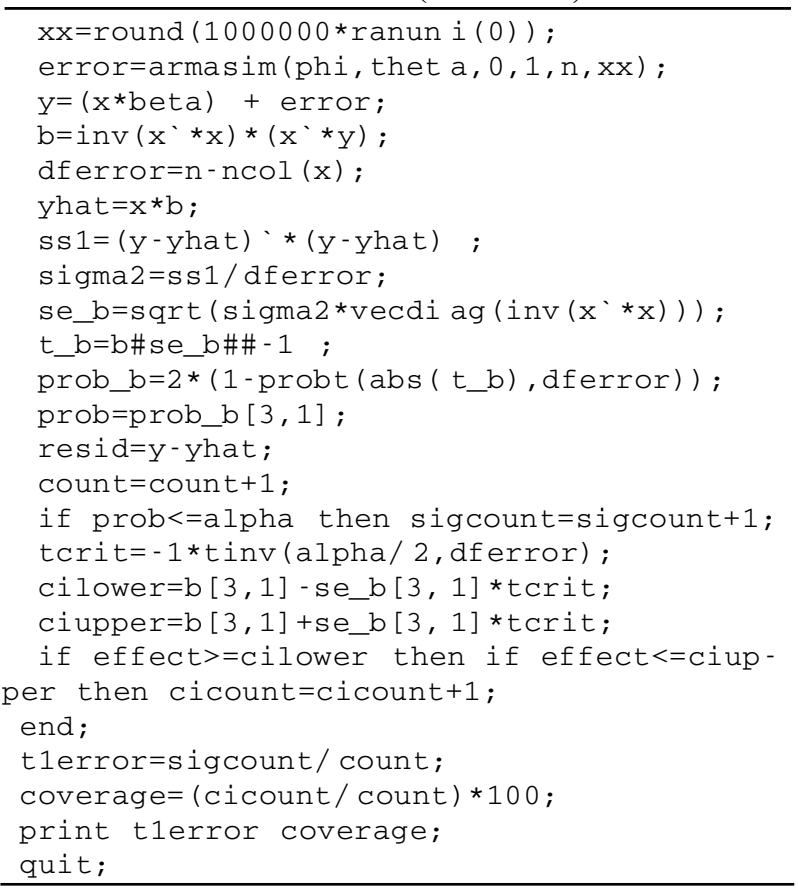

(Manuscript received January 9, 2001; accepted for publication February 28, 2002. . 